Original Research

\title{
Comparison of in vitro biocompatibility and antibacterial activity of two calcium silicate-based materials
}

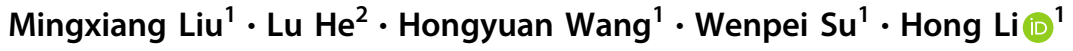

Received: 29 July 2020 / Accepted: 1 April 2021 / Published online: 26 April 2021

(c) The Author(s) 2021

\begin{abstract}
This study is aimed at comparing and evaluating the biocompatibility and antibacterial activities of mineral trioxide aggregate (MTA) and iRoot BP Plus as novel retro-filling materials. Discs of both materials were prepared and incubated for $72 \mathrm{~h}$ to obtain material extracts in medium. Flow cytometry and the 3-(4,5-dimethylthiazol-2-yl)-2,5-diphenyltetrazolium bromide assay were used to assess the rate of apoptosis and proliferation of human periodontal ligament stem cells (hPDLSCs) when exposed to eluates of both materials. The expression levels of alkaline phosphatase, collagen type I, osteocalcin, Runt-related transcription factor-2, and Osterix were tested for evaluating the osteogenic differentiation of hPDLSCs. The antibacterial activities of both materials were compared by the direct contact test. The hPDLSCs stimulated by MTA or iRoot BP Plus eluates showed significantly higher cell viability than that of the control group with no eluates. No significant differences were observed among the percentages of necrotic and apoptotic cells stimulated by MTA and iRoot BP Plus eluates and the control group. The expression of all osteogenic differentiation markers of hPDLSCs in both experimental groups were significantly higher than those of the control group, while the increment values in MTA group were significantly higher than those of the iRoot BP Plus group. The antibacterial activity against Enterococcus faecalis showed no significant difference between MTA and iRoot BP Plus. Therefore, both materials may be suitable for retro-filling applications.
\end{abstract}

Hong Li

endolihong@qq.com

1 Department of Endodontics, Beijing Stomatological Hospital, School of Stomatology, Capital Medical University,

Beijing 100050, China
2 Affiliated Stomatology Hospital of Guangzhou Medical University, School of Stomatology, Guangzhou Medical University, Guangdong 510140, China 


\section{Graphical Abstract}
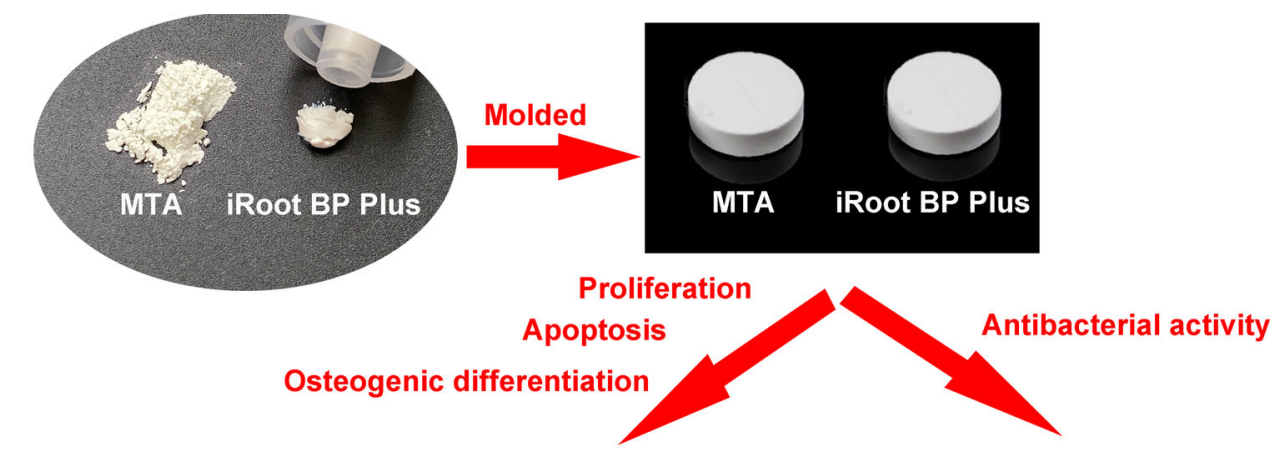

hPDLSCs

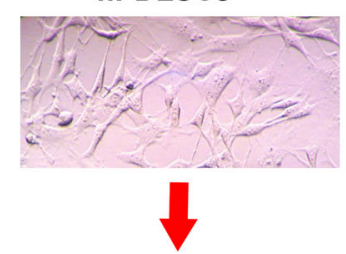

Enterococcus faecalis

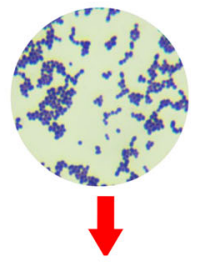

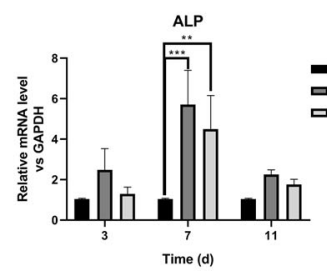
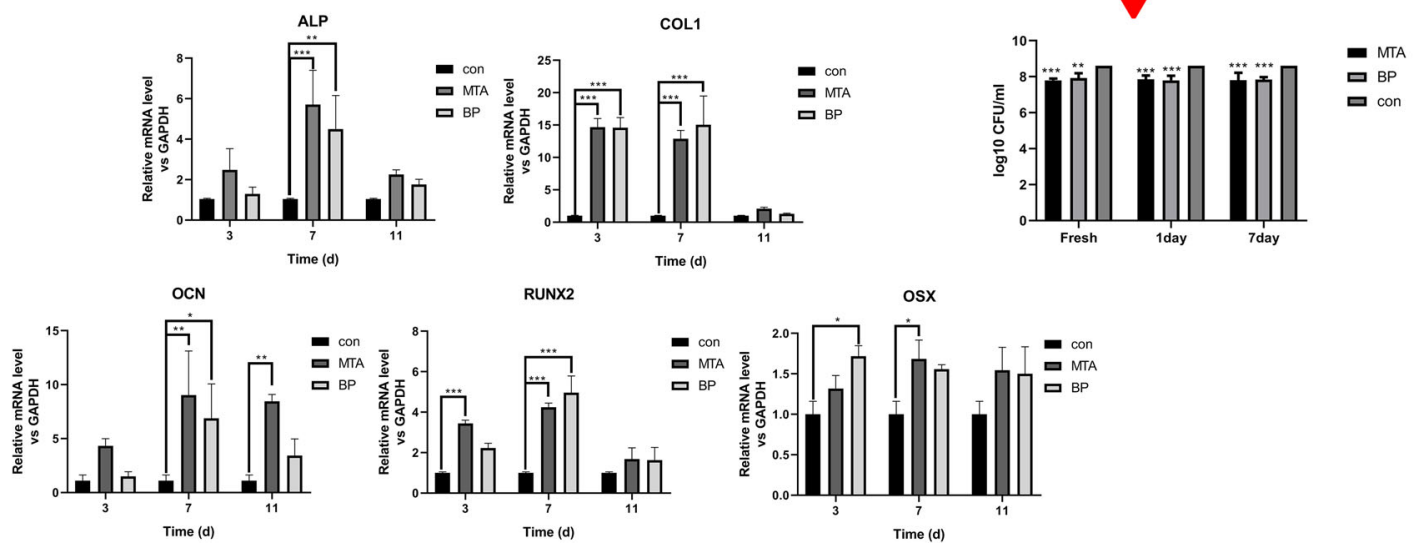

\section{Introduction}

Among various oral biomaterials, mineral trioxide aggregate (MTA) has been widely used in apical surgery and endodontic therapy since the early 1990s because of its outstanding biological and physical properties [1]. MTA is a calcium silicate-based cement composed of tricalcium silicate, tricalcium oxides, tetracalcium aluminoferrite, and so on [2]. It has been successfully used for pulp capping, perforation repairing, root resorption, and apexification [3]. MTA exhibits high $\mathrm{pH}$, good biocompatibility, bioactivity, osteoconductivity, cell viability promotion, and releases calcium hydroxide $\left(\mathrm{Ca}(\mathrm{OH})_{2}\right)$ when exposed to water [1]. It has been shown to stimulate the release of cytokine in osteoblasts, which promotes hard tissue formation [4]. However, a lack of antibacterial activity against Enterococcus faecalis (E.faecalis) was detected using direct contact test (DCT) in a previous study [5]. Due to its high sealing and biocompatibility, MTA is considered the gold standard as a novel root-end filling material [6]. Nevertheless, it has several drawbacks, such as presence of toxic elements in its composition, long setting time, discoloration potential and difficulty in handling; consequently, improvements are necessary [7].

iRoot BP Plus is a new laboratory-synthesized bioceramic material used for oral surgery and permanent root canal repair [8]. iRoot BP Plus is composed of calcium silicate, calcium sulfate, zirconium oxide, calcium phosphate monobasic, and a filler agent [9]. Reports show that iRoot BP Plus is biocompatible, insoluble in water, and produces $\mathrm{Ca}(\mathrm{OH})_{2}$ when in contact with water [10]. Moreover, iRoot BP Plus has demonstrated significant antibacterial activity against Candida albicans and E. faecalis [8]. However, it is reported that iRoot BP Plus significantly decreased cell viability of human osteoblasts as compared to MTA after $48 \mathrm{~h}$ of exposure [6].

Although MTA and iRoot BP Plus have been widely applied clinically, there is still no consensus regarding the biocompatibility and antibacterial activity of the two materials. Insufficient research has been conducted on the antibacterial activity against $E$. faecalis and the osteogenic differentiation 
of human periodontal ligament stem cells (hPDLSCs) of these materials, especially when used as retro-filling materials. Therefore, the purpose of this study was to completely assess the biocompatibility and antibacterial activity of MTA and iRoot BP Plus. The results facilitate a deeper understanding of the underlying mechanisms of the biocompatibility and antibacterial activity of these materials for clinical applications.

\section{Materials and methods}

\subsection{Cement extracts}

MTA (Dentsply Tulsa, OK, USA) and iRoot BP Plus (Innovative Bioceramix, Vancouver, BC, Canada), were prepared according to the manufacturers' instructions. The materials were molded into discs in the plastic molds $(0.8 \mathrm{~cm}$ in diameter and $0.2 \mathrm{~cm}$ in height) under sterile conditions. The discs were placed in a 5\% $\mathrm{CO}_{2}$ and $95 \%$ air atmosphere for $2 \mathrm{~h}$ at $37^{\circ} \mathrm{C}$, washed three times with phosphate-buffered saline (PBS), and subjected to proper ultraviolet irradiation for $30 \mathrm{~min}$. Each material was then socked in aseptic PBS for 2 days; the PBS was replaced every $24 \mathrm{~h}$. Subsequently, the samples were immersed in Dulbecco Modified Eagle's Medium (DMEM) (Gibco Life Technologies, Melbourne, Australia) for $24 \mathrm{~h}$ at $37^{\circ} \mathrm{C}$ in a humid chamber. Finally, the eluates were collected and filtered with $0.22 \mu \mathrm{m}$ filters (Merck Millipore, Billerica, MA, USA). The eluates were diluted at 1:1, 1:2, and 1:4 v/v using fresh DMEM.

\subsection{Viability of hPDLSCs}

The metabolic activity of treated hPDLSCs (ATCC, Manassas, VA, USA) was determined by 3-(4,5-dimethylthiazol-2yl)-2,5-diphenyltetrazolium bromide (MTT Cell Growth Kit, Chemicon, Rosemont, IL, USA). The third generation hPDLSCs were cultured in 96-well plates $\left(1 \times 10^{3}\right.$ cells/well $)$ for $24 \mathrm{~h}$ in a complete medium. Subsequently, the medium was replaced by the diluted eluates $(100 \mu \mathrm{L} /$ well $)$ prepared previously. The control group's cells were cultured in the medium without eluates. At 24 and $48 \mathrm{~h}, 10 \mu \mathrm{L}$ of MTT was added to each well and cells were continuously cultured for $4 \mathrm{~h}$. Then, the supernatant was aspirated and $100 \mu \mathrm{L}$ of dimethyl sulfoxide was added. A microplate reader (VersaMax Microplate reader, Sunnyvale, CA, USA) was used to measure the absorbance at $490 \mathrm{~nm}$. The relative proliferation rate was calculated as (assay group/control group) $* 100 \%$.

\subsection{Analysis of apoptosis and necrosis by flow cytometry}

Cell apoptosis and necrosis was evaluated using Annexin V$\mathrm{PE} / 7-\mathrm{AAD}$ staining [11]. The third generation hPDLSCs incubated with various eluates (undiluted) for $72 \mathrm{~h}$ were collected with centrifugation, and resuspended to $2 \times 10^{5}$ cells/ $\mathrm{mL}$. The cells were stained with PE conjugated annexin- $\mathrm{V}$ and 7-AAD (Immunostep, Salamanca, Spain), according to the flow cytometry protocol for a viability assay. Briefly, $1 \mu \mathrm{g} / \mathrm{mL}$ 7-AAD fluorescent dye was added to the cell suspensions, incubated for $10 \mathrm{~min}$ at $37^{\circ} \mathrm{C}$, and centrifuged for $5 \mathrm{~min}$ at $800 \mathrm{rpm}$ and $4{ }^{\circ} \mathrm{C}$. The supernatant was removed; $1 \mathrm{~mL}$ of PE dye solution was added to the cells, stained in the dark for $15 \mathrm{~min}$, and then filtered. The stained cells were analyzed using a ACEA NovoCyte flow cytometer (Agilent, Lexington, MA, USA). Percentages of live, early, late apoptotic, and necrotic cells were determined by the flow cytometer by using cells cultured in medium without any experimental eluate as control.

\subsection{In vitro osteogenic differentiation assay}

The third generation hPDLSCs were cultured in six-well plates $\left(1 \times 10^{5}\right.$ cells/well $)$ placed in an incubator for $24 \mathrm{~h}$ at $37^{\circ} \mathrm{C}$ and $5 \% \mathrm{CO}_{2}$. Then, the medium was replaced by the extraction medium prepared previously. Cells cultured in medium without any experimental eluate were used as the control. The eluates changed every 3 days. After 3, 7, and 11 days, cells and supernatant were collected for subsequent tests.

\subsubsection{Real-time reverse transcription polymerase chain reaction (real-time RT-PCR)}

Real-time RT-PCR was used to quantify messenger RNA (mRNA) levels of alkaline phosphatase (ALP), collagen type I (COL1), osteocalcin (OCN), Runt-related transcription factor-2 (RUNX2), and Osterix(OSX). Total RNA was isolated from each culture using the RNEasy kit (Qiagen, Valencia, CA, USA) and used as a template for the reversetranscriptase reaction. The cDNAs were used for templates for real-time RT-PCR. The ALP, COL1, OCN, RUNX2, and OSX gene sequences of hPDLSCs were retrievable from the NCBI database, and utilized for constructing their respective upstream and downstream fragment primer pairs (Table 1). Glyceraldehyde-3-phosphate dehydrogenase (GAPDH) was included as an internal reference gene. The SYBR Green PCR kit (Qiagen) was used to perform realtime RT-PCR. The Ct value indicated the number of PCR cycles needed to gain a certain level of fluorescence. The data were calculated using formula $2^{(-\Delta \Delta \mathrm{Ct})}$.

\subsubsection{Western blot analysis}

The total protein was extracted using an RIPA lysis buffer (Millipore, Billerica, MA, USA). The protein concentration was tested by the bicinchoninic acid Protein Assay Kit (BioRad, Berkeley, Canada). Proteins were separated by $10 \%$ 
Table 1 List of primers used for real-time RT-PCR

\begin{tabular}{llll}
\hline Genes & Primers & Sequence $\left(5^{\prime}-3^{\prime}\right)$ & dsDNA \\
\hline ALP & Forward & AGAATCAGAACCACAGGACGGG & 22 \\
& Reverse & TTCAAGTCACCTGGGCAAATG & 21 \\
COL1 & Forward & GCGAGAGCATGACCGATGGATTC & 23 \\
& Reverse & GCCTTCTTGAGGTTGCCAGTCTG & 23 \\
OCN & Forward & CCCTCACACTCCTCGCCCTATT & 22 \\
& Reverse & GGTCAGCCAACTCGTCACAGTC & 22 \\
Osterix & Forward & ATCCAGCCCCCTTTACAAGC & 20 \\
& Reverse & TAGCATAGCCTGAGGTGGGT & 20 \\
RUNX2 & Forward & ACCAGCAGCACTCCATATCTCTACT & 25 \\
& Reverse & CTTCCATCAGCGTCAACACCATCA & 24 \\
GAPDH & Forward & TCAAGAAGGTGGTGAAGCAGG & 21 \\
& Reverse & GCGTCAAAGGTGGAGGAGTG & 20 \\
\hline
\end{tabular}

dodecyl sulfate, sodium salt-Polyacrylamide gel electrophoresis (PAGE) and then transferred to polyvinylidene fluoride membranes (Millipore) which were then blocked with $5 \%$ fat-free milk at room temperature for $2 \mathrm{~h}$ before incubating overnight with primary antibodies against ALP, COL1, OCN, RUNX2, OSX, and GAPDH (Abcam, Cambridge, UK) at $4{ }^{\circ} \mathrm{C}$. After washing for 3 times, the membranes were incubated in corresponding secondary antibodies for $1 \mathrm{~h}$. The blotted bands were detected using an enhanced chemiluminescence detection kit (Amersham Pharmacia Biotech, Little Chalfont, UK).

\subsection{Antibacterial activity}

The antimicrobial activity of the test materials was evaluated by the DCT against E. faecalis ATCC 29212 (Manassas). The strains were grown in tryptic soy agar (TSA, Merc, Germany) at $37^{\circ} \mathrm{C}$ for $18-24 \mathrm{~h}$ in a $5 \% \mathrm{CO}_{2}$ and $95 \%$ air atmosphere, and collected for subsequent experiments.

A total of $0.05 \mathrm{~g}$ MTA or iRoot BP Plus was evenly coated onto the bottoms of the wells of a 96-well plate. Each material was coated with 9 wells, which were divided into three groups with three wells in each group. Samples tested at $20 \mathrm{~min}, 1$ day, and 7 days after mixing (MTA) or addition of the sterile distilled water (iRoot BP Plus) were designated as "fresh group", "1 day group", and "7 day group", respectively [8, 12]. A $10 \mu \mathrm{L}$ E. faecalis suspension (approximately $1 \times 10^{6}$ cells) was added to each well. After incubation in a moist atmosphere at $37^{\circ} \mathrm{C}$ for $1 \mathrm{~h}, 100 \mu \mathrm{L}$ tryptic soy broth (TSB, Merc) was added to each well. The solutions were mixed for $1 \mathrm{~min}$, the obtained microbial suspensions were diluted tenfold in TSB to $10^{-3}$. After incubation at $37^{\circ} \mathrm{C}$ for $48 \mathrm{~h}$, the number of colonies were counted and expressed as $\log 10$ values. E. faecalis suspensions added to wells without any material were used as control.

\subsection{Statistical analysis}

The values were expressed as the mean \pm standard error of three independent experiments. Statistically significant differences were assessed by two-way analysis of variance using the GraphPad Prism 8.0.2 software (GraphPad Prism Software, La Jolla, CA). Values of $p<0.05$ were considered as statistically significantly different.

\section{Results}

\subsection{Proliferation of hPDLSCs in the presence of different eluates}

To determine the influences of both materials about the proliferation of hPDLSCs, the cell viability of hPDLSCs in the eluates of the two materials was measured with the MTT assay (Fig. 1a, b). hPDLSCs of MTA and iRoot BP Plus (dilution 1:1) showed significantly higher cell viability at $24 \mathrm{~h}$ and $48 \mathrm{~h}$ than that of the control group $(* p<0.05$, $* * * p<0.001)$. However, 1:2 and 1:4 dilutions of iRoot BP Plus had an insignificant effect on cell viability (Fig. 1b). Both materials with 1:1 dilutions showed higher values than those with 1:2 and 1:4 dilutions. At $24 \mathrm{~h}$, the cell viability of MTA was significantly higher than that of the iRoot BP Plus group for all dilutions $(* p<0.05)$; however, no significant difference between both materials was observed at $48 \mathrm{~h}(p>0.05)$.

\subsection{Apoptosis and necrosis of hPDLSCs in the presence of different eluates}

The two-dimensional dot plots shown in Fig. 1c-e exhibited the distribution of live (Annexin- $\mathrm{V}^{-} / 7-\mathrm{AAD}^{-}$), early $\left(\right.$ Annexin- $\mathrm{V}^{+} / 7-\mathrm{AAD}^{-}$), late apoptotic (Annexin- $\mathrm{V}^{+} / 7$ $\mathrm{AAD}^{+}$and necrotic cells (Annexin- $\left.\mathrm{V}^{-} / 7-\mathrm{AAD}^{+}\right)$in 

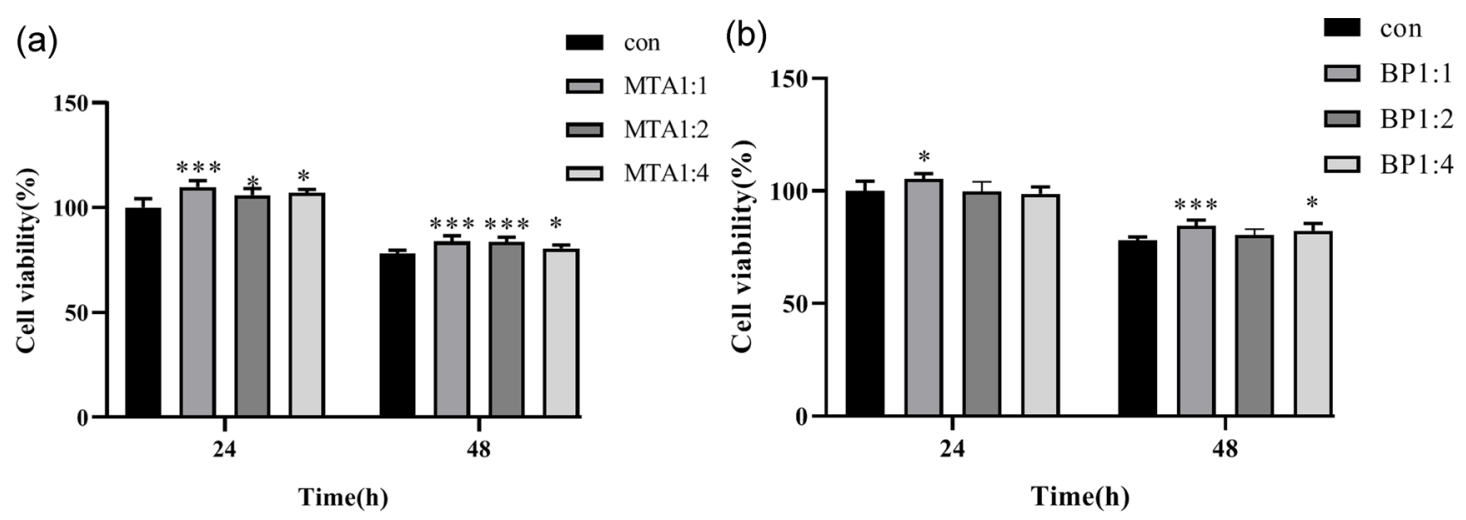

(c)

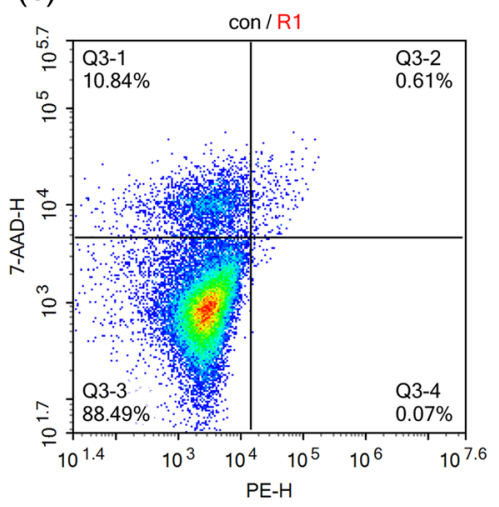

(d)

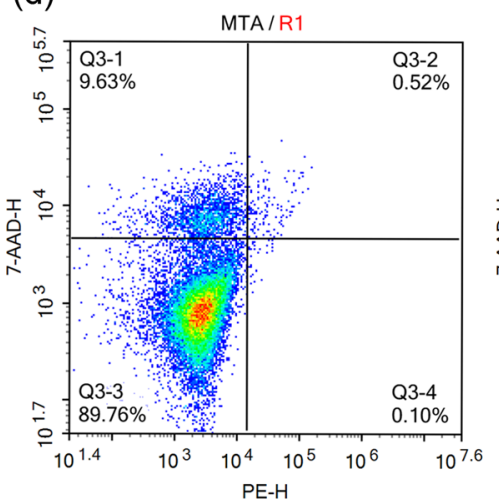

(e)

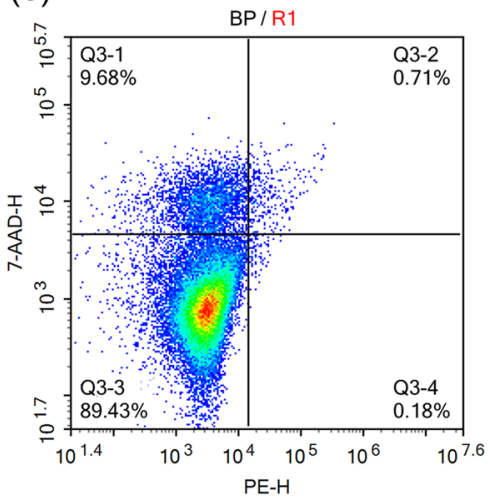

Fig. 1 Viability rate of hPDLSCs in different eluates in vitro by MTT assay. a hPDLSCs cultured in MTA eluate for 24 and $48 \mathrm{~h}$; b hPDLSCs cultured in iRoot BP Plus eluate for 24 and 48 h. $\left({ }^{*} p<0.05\right.$, $* * p<0.01$, and $* * * p<0.001 ; n=5)$. Representative two-dimensional dot plots images of the flow cytometry data from Annexin V-PE and 7-
AAD stained hPDLSCs in: c control; d MTA; and e iRoot BP Plus. The dot plots in the three images indicate the distribution of life (lower left), early (lower right) or late apoptotic (upper right), and necrotic cells (upper left)
hPDLSCs that were exposed to the eluates of tested materials. The percentage of live cells cultured for MTA and iRoot BP Plus eluates after $72 \mathrm{~h}(>89 \%)$ were slightly higher than that of the control group (88.49\%), although this difference was not statistically significant $(p>0.05)$. The percentage of necrotic and apoptotic cells with both materials were similar to those of the control.

\subsection{In vitro osteogenic differentiation assay}

\subsubsection{Real-time RT-PCR analysis}

To investigate the ability of osteogenic differentiation of hPDLSCs treated with root-end filling materials, the levels of mRNA expression of the osteogenic differentiation markers (ALP, COL1, OCN, RUNX2, and OSX) were assessed (Fig. 2). On day 7, the mRNA expression of ALP in both MTA and iRoot BP Plus groups was higher than those on day 3 and day 11 , and significantly higher than those in the control group on day $7(* * p<0.01, * * * p<0.001)$. Meanwhile, the mRNA expression of COL1 in the two groups on day 3 and day 7 were higher than those in the control group
$(* * * P<0.001)$, although no significant difference compared with control group on day 11 were observed. On day 7 , both experiment groups exhibited significantly higher mRNA expressions of OCN and RUNX2 than those of the control group $(* p<0.05, * * p<0.01, * * * p<0.001)$.

The mRNA level of OSX in the iRoot BP Plus group was significantly higher than that of the control group on day 3 $\left({ }^{*} p<0.05\right)$. Although, on day 7 , a significantly higher OSX mRNA expression was observed in the MTA group than that of the control group $\left({ }^{*} p<0.05\right)$. However, mRNA levels of all genes showed insignificant difference between MTA and iRoot BP Plus groups throughout the studied timeframe.

\subsubsection{Western blot analysis}

The Western blot assay was used to investigate the protein expression levels of the osteogenic differentiation markers ALP, COL1, OCN, RUNX2, and OSX (Fig. 3). Western blot analysis exhibited that the protein expression of all markers of hPDLSCs in both MTA and iRoot BP Plus groups were significantly higher than those of the control group on days 3,7 , and $11(* * p<0.01, * * * p<0.001)$, 


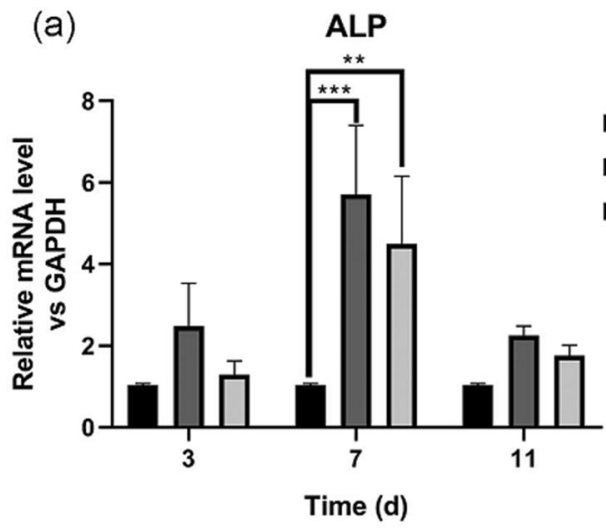

(c)

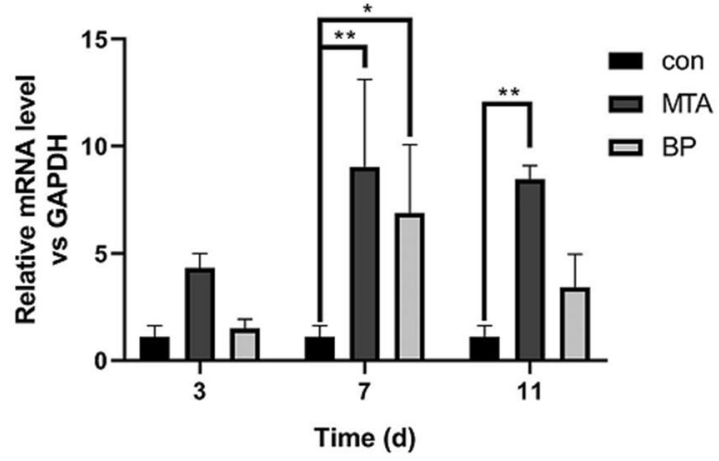

(e)

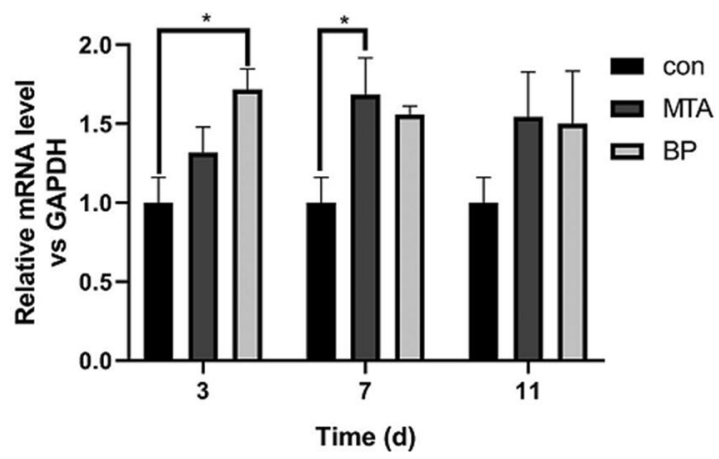

Fig. 2 mRNA expression levels of ALP (a), COL1 (b), OCN (c), RUNX2 (d), and OSX (e) of hPDLSCs with extracts of MTA and

except for the marker ALP on day 3 (Fig. 3b). The protein expression values of all markers in the MTA groups were significantly higher than those in the iRoot BP Plus $\left({ }^{*} p<\right.$ $0.05, * * * p<0.001$ ), except for COL1 on day 11 (Fig. $3 \mathrm{c}$ ). On day 7, the protein expression of ALP and OCN in both groups were significantly higher than that on day $3(* * * p<$ 0.001). On the contrary, the expression of COL1 in the MTA and iRoot BP Plus groups showed a significant reduction on day $11(* * * p<0.001)$. (b)

COL1

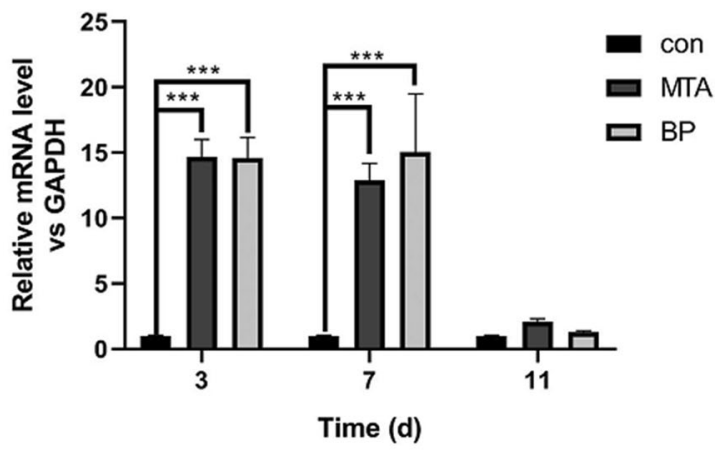

(d)

RUNX2

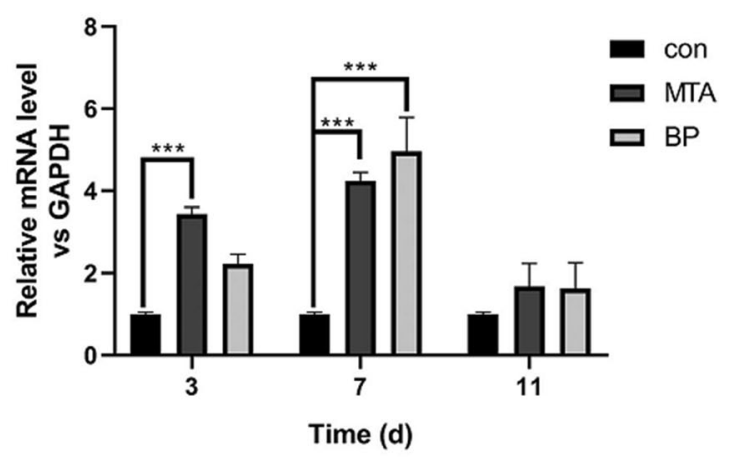

iRoot BP Plus, determined by real-time RT-PCR at three time-points (day 3,7 , and 11). $(* p<0.05, * * p<0.01$, and $* * * p<0.001 ; n=5$ )

\subsection{Antibacterial activity of MTA and iRoot BP plus against $E$. faecalis}

The results of the DCT for the the fresh, 1 day and 7 day groups with E. faecalis are shown in Fig. 4. All MTA and iRoot BP Plus groups indicated similar inhibition of $E$. faecalis and statistically significant differences were observed compared with those of the control $(* * p<0.01$, $* * * p<0.001)$. 
(a)

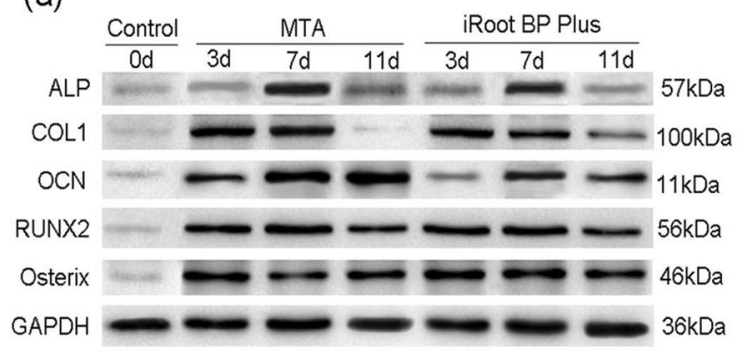

(c)

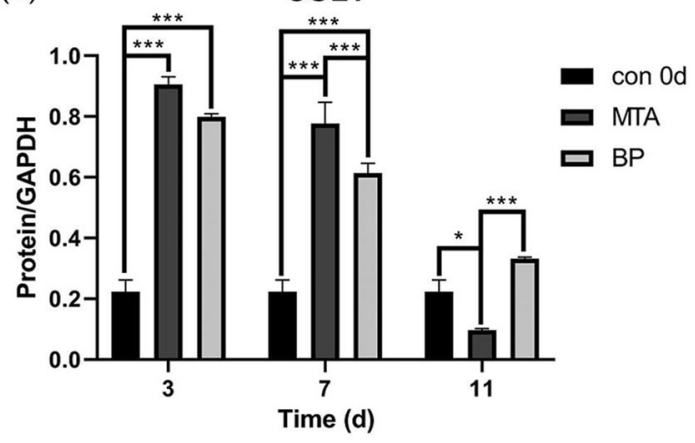

(e)

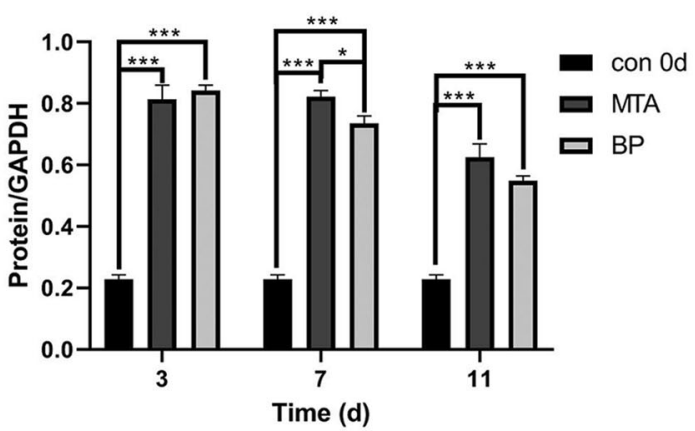

Fig. 3 a Protein expression levels of ALP, COL1, OCN, RUNX2, and OSX of hPDLSCs with extracts of MTA and iRoot BP Plus, determined by Western blot at three different time-points (day 3, 7 and 11).

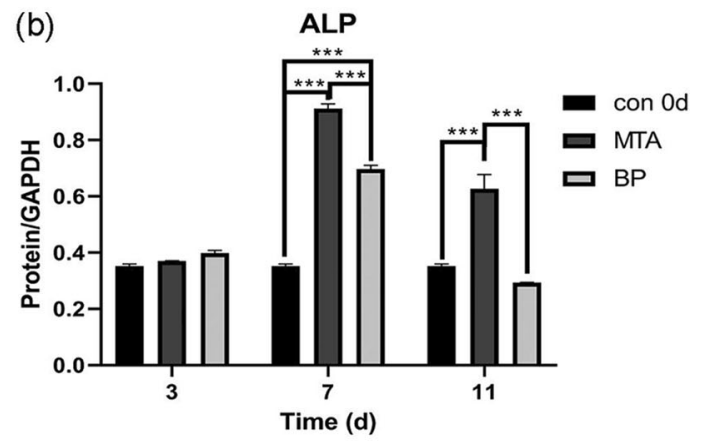

(d)

OCN

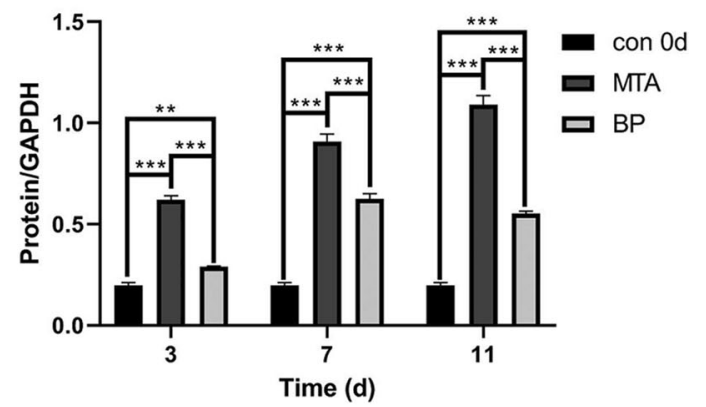

(f)

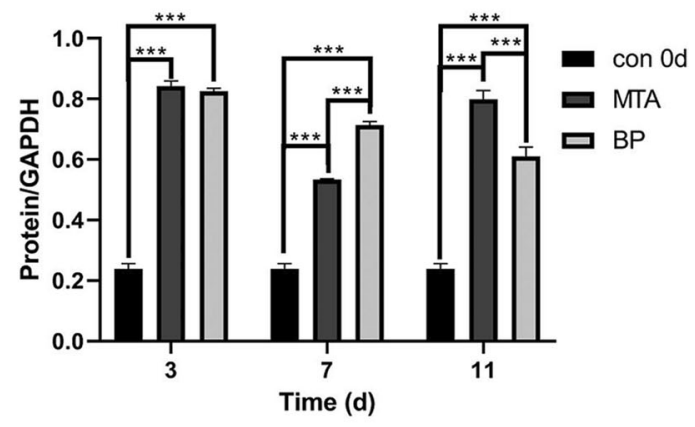

GAPDH served as an internal control. b-f Grayscale analysis of $\mathbf{a}$. $\left({ }^{*} p<0.05, * * p<0.01\right.$, and $\left.{ }^{* * *} p<0.001 ; n=5\right)$

\section{Discussion}

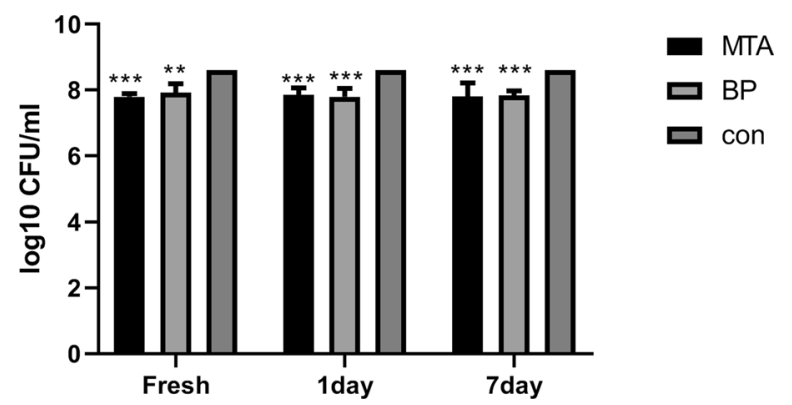

Fig. 4 Survival of E. faecalis after incubation with MTA and iRoot BP Plus at different experimental periods using the direct contact test. $(* * p<0.01$, and $* * * p<0.001 ; n=5)$
A root-end filling material should have the highest biocompatibility because it is in close contact with periapical tissues [13]. The interactions between the stem cells of periapical tissues and root-end filling materials have been considered as important factors because they promote healing [14]. The ability of these cells to form cementum, alveolar bone, periodontal ligaments, nerves, and blood vessels has been demonstrated successfully in vivo [15]. It is widely recognized the interactions between dental stem cells and bioactive materials are very significant in root-end filling applications [16]. Therefore, hPDLSCs were used 
herein to simulate the close proximity between the tested materials and the apical and periodontal tissues.

In this study, cellular analysis was performed using the eluates from both tested materials. According to the International Standard ISO 10993-12 Biological Evaluation of Medical Devices [17], we obtained eluates of the materials, and hPDLSCs were treated with different eluates, similar to previously reported studies [18-20]. The tests performed on those eluates allowed both qualitative and quantitative assessment of biocompatibility. It could simulate the clinical conditions: the interaction between hPDLSCs and retrofilling materials in blood clots and body fluids.

In vitro cytotoxicity assays are suitable to evaluate basic biological aspects relative to biocompatibility and are simple, reproducible, and cost-effective [21]. Although the results of the MTT assay varied according to dilution and time, both materials could significantly improve the viability of hPDLSCs, showing similar cell compatibility. This result was in good agreement with a previous report on iRoot BP biocompatibility [10]. However, this was not consistent with the results obtained by Zhang et al. [22]; they described the proliferation suppression of human dental pulp cells (hDPCs) in the MTA group during the whole intervention time periods. This discrepancy may be explained because the materials used by Zhang et al. [22] were placed directly in contact with hDPCs, rather than intervening through the material eluates. The results of flow cytometry analyses revealed that neither MTA nor iRoot BP Plus induced apoptosis, thus preserving cell viability. This was consistent with previous reports describing negligible in vitro cytotoxicity of Pro Root MTA and iRoot BP Plus [23].

Periapical bone tissue defects caused by chronic periapical periodontitis can be healed by endodontic therapy or apical surgery [24]. Therefore, the root-end filling materials must have an excellent capability to promote the regeneration of hard tissues. To explore the influences of both materials on the osteogenic differentiation of hPDLSCs, a series of genes and proteins related to osteogenesis were used in this study. ALP is a representative marker of osteoblasts that directly reflects their activity; it is often used as a marker of early osteogenic differentiation and mineralization [25]. OCN is a noncollagenous protein, which regulates the mineralization of hard tissues; it is considered as a marker of terminal differentiation in bone regeneration [26]. RUNX2, involving all stages of biomineralization, is up-regulated in the early stages and downregulated during the terminal phase and it is also active in mature osteoblasts [27, 28]. OSX is a downstream signaling gene of RUNX2, which is an important transcription factor in osteoblast differentiation [25]. Consequently, the ratio of RUNX2-to-OSX is often considered as an early osteogenic differentiation marker. For the two materials evaluated in this study, the mRNA expression of ALP, RUNX2, OCN, and OSX were upregulated, which was similar with the results obtained by Lu et al. [29]. Meanwhile, we found that the MTA group exhibited significantly higher protein expressions of ALP, RUNX2, OCN, and OSX than those of the iRoot BP Plus group. These data indicated that both materials triggered osteogenic differentiation of hPDLSCs, with MTA exhibiting a superior capacity. Our results indicated the expression of COL1 was downregulated on day 11 for both materials groups. Widbiller et al. [30] reported that COL1, as an early related marker, was down-regulated during the odontoblast maturation process. Hence, we hypothesize that both materials can also induce odontogenic differentiation of hPDLSCs. However, more genes related to odontogenic differentiation need to be tested to confirm this hypothesis.

E. faecalis is one of the most detected microorganisms in persistent apical periodontitis [31]. This gram-positive facultative anaerobe exists inside the root canal, and is related to periradicular lesions; it can penetrate and colonize dentinal tubules [32]. For these reasons, E. faecalis was selected in this study to evaluate the antimicrobial activity of the materials tested.

For antimicrobial analysis, the direct contact test we performed is a quantitative and reproducible assay that relies on the direct contact of the test microorganisms with test material for a controlled period time; further, this test is independent of the diffusion and solubility properties of the material tested and media [5]. In addition, this method allows the exact measurement of surviving bacteria. The direct contact test can simulate the contact of microorganisms with retro-filling materials in the retrograde cavity of resected roots. Both materials showed significant and similar antimicrobial activities. This result was in good agreement with the conclusions of other studies [8, 33]. Both MTA and iRoot BP Plus produce a calcium silicate hydrogel and $\mathrm{Ca}(\mathrm{OH})_{2}$ when in contact with water of the dentin and periapical tissues [8]. Scientific reports indicate $\mathrm{Ca}(\mathrm{OH})_{2}$ alters the wholeness of cytomembrane and the transport of bacteria nutrients [34]. Additionally, the alkalizing effect of $\mathrm{Ca}(\mathrm{OH})_{2}$ could inhibit significantly the bacterial normal metabolism, growth, and cellular division [35]. Moreover, antimicrobial activity of zirconium oxide against both gram-positive bacteria via redox reactions has been reported in literature [36, 37]. Although several studies have reported the antimicrobial activity of MTA, the results are not consistent $[5,38]$. This might be attributed to the use of different experimental methods, bacterial strains, and aerobic or anaerobic culture conditions.

\section{Conclusion}

The novel ready-to-use root-end filling material, iRoot BP Plus exhibited excellent in vitro biocompatibility with 
hPDLSCs because it promoted proliferation of cells without inducing apoptosis. Both MTA and iRoot BP Plus showed significant as well as similar antibacterial activity against $E$. faecalis. Moreover, both materials could significantly improve the osteogenic differentiation of hPDLSCs. Therefore, both calcium silicate-based materials could be promising root-end filling materials. We believe that further in vivo investigation of these oral biomaterials is required in the future.

Acknowledgements We would like to thank Mr. Benxiang Hou and Mr. Deqiang Zheng for their assistance with the experimental methods and statistical analysis, respectively. All data presented in this paper have independently been generated and interpreted by the authors. This study was funded by the National Natural Science Foundation of China (grant number 81500833).

\section{Compliance with ethical standards}

Conflict of interest The authors declare no competing interests.

Publisher's note Springer Nature remains neutral with regard to jurisdictional claims in published maps and institutional affiliations.

Open Access This article is licensed under a Creative Commons Attribution 4.0 International License, which permits use, sharing, adaptation, distribution and reproduction in any medium or format, as long as you give appropriate credit to the original author(s) and the source, provide a link to the Creative Commons license, and indicate if changes were made. The images or other third party material in this article are included in the article's Creative Commons license, unless indicated otherwise in a credit line to the material. If material is not included in the article's Creative Commons license and your intended use is not permitted by statutory regulation or exceeds the permitted use, you will need to obtain permission directly from the copyright holder. To view a copy of this license, visit http://creativecommons. org/licenses/by/4.0/.

\section{References}

1. Zhou HM, Du TF, Shen Y, Wang ZJ, Zheng YF, Haapasalo M. In vitro cytotoxicity of calcium silicate-containing endodontic sealers. J Endod. 2015;41:56-61.

2. Solanki NP, Venkappa KK, Shah NC. Biocompatibility and sealing ability of mineral trioxide aggregate and biodentine as root-end filling material: a systematic review. J Conserv Dent. 2018;21:10-15.

3. Ciasca M, Aminoshariae A, Jin G, Montagnese T, Mickel A. A comparison of the cytotoxicity and proinflammatory cytokine production of EndoSequence root repair material and ProRoot mineral trioxide aggregate in human osteoblast cell culture using reversetranscriptase polymerase chain reaction. J Endod. 2012;38:486-9.

4. Torabinejad M, Parirokh M. Mineral trioxide aggregate: a comprehensive literature review-part II: leakage and biocompatibility investigations. J Endod. 2010;36:190-202.

5. Morgental RD, Vier-Pelisser FV, Oliveira SD, Antunes FC, Cogo DM, Kopper PM. Antibacterial activity of two MTA-based root canal sealers. Int Endod J. 2011;44:1128-33.

6. De-Deus G, Canabarro A, Alves GG, Marins JR, Linhares AB, Granjeiro JM. Cytocompatibility of the ready-to-use bioceramic putty repair cement iRoot BP Plus with primary human osteoblasts. Int Endod J 2012;45:508-13.

7. Amezcua O, Gonzalez ÁC, Borges ÁH, Bandeca MC, Estrela CR, Estrela C. Sealing ability of root-end filling materials. J Contemp Dent Pract. 2015;16:210-4.

8. Damlar I, Ozcan E, Yula E, Yalcin M, Celik S. Antimicrobial effects of several calcium silicate-based root-end filling materials. Dent Mater J. 2014;33:453-7.

9. Liu S, Wang S, Dong Y. Evaluation of a bioceramic as a pulp capping agent in vitro and in vivo. J Endod. 2015;41:652-7.

10. Öncel Torun Z, Torun D, Demirkaya K, Yavuz ST, Elçi MP, Sarper M, et al. Effects of iRoot BP and white mineral trioxide aggregate on cell viability and the expression of genes associated with mineralization. Int Endod J. 2015;48:986-93.

11. Collado-González M, López-García S, García-Bernal D, OñateSánchez RE, Tomás-Catalá CJ, Moraleda JM, et al. Biological effects of acid-eroded MTA Repair HP and ProRoot MTA on human periodontal ligament stem cells. Clin Oral Investig 2019;23:3915-24.

12. Lovato KF, Sedgley CM. Antibacterial activity of endosequence root repair material and proroot MTA against clinical isolates of Enterococcus faecalis. J Endod. 2011;37:1542-6.

13. Ma J, Shen Y, Stojicic S, Haapasalo M. Biocompatibility of two novel root repair materials. J Endod. 2011;37:793-8.

14. Sultana N, Singh M, Nawal RR, Chaudhry S, Yadav S, Mohanty $\mathrm{S}$, et al. Evaluation of biocompatibility and osteogenic potential of tricalcium silicate-based cements using human bone marrowderived mesenchymal stem cells. J Endod. 2018;44:446-51.

15. Vera-Sánchez M, Aznar-Cervantes S, Jover E, García-Bernal D, Oñate-Sánchez RE, Hernández-Romero D, et al. Silk-fibroin and graphene oxide composites promote human periodontal ligament stem cell spontaneous differentiation into osteo/cementoblast-like cells. Stem Cells Dev. 2016;25:1742-54.

16. Wang Y, Zhou Y, Jin L, Pang X, Lu Y, Wang Z, et al. Mineral trioxide aggregate enhances the osteogenic capacity of periodontal ligament stem cells via NF- $\mathrm{BB}$ and MAPK signaling pathways. J Cell Physiol. 2018;233:2386-97.

17. ISO, 10993 biological evaluation of medical devices. Part 12: Sample preparation and reference materials. Switzerland, 2012.

18. Saeed MA, El-Rahman MA, Helal ME, Zaher AR, Grawish ME. Efficacy of human platelet rich fibrin exudate vs fetal bovine serum on proliferation and differentiation of dental pulp stem cells. Int J Stem Cells. 2017;10:38-47.

19. Qiu W, Sun B, He F, Zhang Y. MTA-induced Notch activation enhances the proliferation of human dental pulp cells by inhibiting autophagic flux. Int Endod J. 2017;50:e52-e62.

20. Dou L, Yan Q, Yang D. Effect of five dental pulp capping agents on cell proliferation, viability, apoptosis and mineralization of human dental pulp cells. Exp Ther Med. 2020;19:2377-83.

21. De-Deus G, Canabarro A, Alves G, Linhares A, Senne MI, Granjeiro JM. Optimal cytocompatibility of a bioceramic nanoparticulate cement in primary human mesenchymal cells. J Endod. 2009;35:1387-90.

22. Zhang S, Yang X, Fan M. Bioaggregate and iRoot BP Plus optimize the proliferation and mineralization ability of human dental pulp cells. Int Endod J. 2013;46:923-9.

23. Jiang Y, Zheng Q, Zhou X, Gao Y, Huang D. A comparative study on root canal repair materials: a cytocompatibility assessment in L929 and MG63 cells. Sci World J. 2014;2014:463826.

24. Tsesis I, Rosen E, Taschieri S, Telishevsky SY, Ceresoli V, Del FM. Outcomes of surgical endodontic treatment performed by a modern technique: an updated meta-analysis of the literature. J Endod. 2013;39:332-9.

25. Li Z, Wang W, Xu H, Ning Y, Fang W, Liao W, et al. Effects of altered CXCL12/CXCR4 axis on BMP2/Smad/Runx2/Osterix axis and osteogenic gene expressions during osteogenic differentiation of MSCs. Am J Transl Res. 2017;9:1680-93. 
26. Rathinam E, Rajasekharan S, Chitturi RT, Declercq H, Martens L, De Coster P. Gene expression profiling and molecular signaling of various cells in response to tricalcium silicate cements: a systematic review. J Endod. 2016;42:1713-25.

27. Camilleri S, McDonald F. Runx2 and dental development. Eur J Oral Sci. 2006;114:361-73.

28. Ducy P, Starbuck M, Priemel M, Shen J, Pinero G, Geoffroy V, et al. A Cbfa1-dependent genetic pathway controls bone formation beyond embryonic development. Genes Dev. 1999;13:1025-36.

29. Lu J, Li Z, Wu X, Chen Y, Yan M, Ge X, et al. Plus promotes osteo/odontogenic differentiation of bone marrow mesenchymal stem cells via MAPK pathways and autophagy. Stem Cell Res Ther. 2019;10:222.

30. Widbiller M, Lindner SR, Buchalla W, Eidt A, Hiller K-A, Schmalz G, et al. Three-dimensional culture of dental pulp stem cells in direct contact to tricalcium silicate cements. Clin Oral Investig. 2016;20:237-46.

31. Taschieri S, Bettach R, Lolato A, Moneghini L, Fabbro MD. Endodontic surgery failure: SEM analysis of root-end filling. J Oral Sci. 2011;53:393-6.

32. Pourhajibagher M, Kazemian H, Chiniforush N, Hosseini N, Pourakbari B, Azizollahi A, et al. Exploring different photosensitizers to optimize elimination of planktonic and biofilm forms of Enter- ococcus faecalis from infected root canal during antimicrobial photodynamic therapy. Photodiagn Photodyn Ther. 2018;24:206-11.

33. ElReash AA, Hamama H, Eldars W, Lingwei G, Zaen El-Din $\mathrm{AM}$, Xiaoli X. Antimicrobial activity and $\mathrm{pH}$ measurement of calcium silicate cements versus new bioactive resin composite restorative material. BMC Oral Health. 2019;19:235.

34. Dianat O, Saedi S, Kazem M, Alam M. Antimicrobial activity of nanoparticle calcium hydroxide against Enterococcus faecalis: an in vitro study. Iran Endod J. 2015;10:39-43.

35. Pacios MG, Silva C, López ME, Cecilia M. Antibacterial action of calcium hydroxide vehicles and calcium hydroxide pastes. J Investig Clin Dent. 2012;3:264-70.

36. Mftah A, Alhassan FH, Al-Qubaisi MS, El Zowalaty ME, Webster TJ, Sh-Eldin M, et al. Physicochemical properties, cytotoxicity, and antimicrobial activity of sulphated zirconia nanoparticles. Int J Nanomed 2015;10:765-74.

37. Rtimi S, Pulgarin C, Sanjines R, Nadtochenko V, Lavanchy JC, Kiwi J. Preparation and mechanism of Cu-decorated $\mathrm{TiO}_{2}-\mathrm{ZrO}_{2}$ films showing accelerated bacterial inactivation. ACS Appl Mater Interfaces. 2015;7:12832-9.

38. Parirokh M, Torabinejad M. Mineral trioxide aggregate: a comprehensive literature review-part I: chemical, physical, and antibacterial properties. J Endod. 2010;36:16-27. 